\title{
MONOTONICITY OF THE ZEROS OF THE THIRD DERIVATIVE OF BESSEL FUNCTIONS
}

\author{
Lee Lorch and Peter Szego
}

\begin{abstract}
It is shown that $j_{\nu k}^{\prime \prime \prime}$ is an increasing function of $\nu, 1<\nu<\infty$, for each fixed $k=1,2, \ldots$, and also that this holds in $0<\nu<\infty$ when $j_{\nu k}^{\prime \prime \prime} \geq \sqrt{3}$. Here $j_{\nu k}^{\prime \prime \prime}$ is the $k$-th positive zero of $J_{\nu}^{\prime \prime \prime}(x)$, the third derivative of the Bessel function of first kind and order $\nu$. These results follow from a representation derived for $d j_{\nu k}^{\prime \prime \prime} / d \nu, \nu>0$. In addition, a number of inequalities for $j_{\nu k}^{\prime \prime \prime}$ are established, especially for $k=1$.
\end{abstract}

\section{Introduction}

We prove here that the $k$-th positive zero, $j_{\nu k}^{\prime \prime \prime}$, of $J_{\nu}^{\prime \prime \prime}(x)$, the third derivative of the Bessel function of order $\nu$, is an increasing function of the order $\nu$, for each fixed $k=1,2,3, \ldots$, for $1 \leq \nu<\infty$. This is shown to be true as well for $0<\nu<1$ for these $j_{\nu k}^{\prime \prime \prime} \geq \sqrt{3}$, in particular for all the zeros of $J_{\nu}^{\prime \prime \prime}(x)$ which occur past the first arch of $J_{\nu}(x)$, since $j_{\nu 1}>j_{01}=2.404 \ldots>\sqrt{3}, \nu>0,[10, \S 15.6(2)$, p. 508].

The behaviour of those $j_{\nu k}^{\prime \prime \prime}<\sqrt{3}$, where they exist when $0<\nu<1$, is more complicated. Their nature is depicted in graphics kindly prepared by Alfred Gray of the University of Maryland using Mathematica. These suggest that such zeros exist when and only when $\nu_{3}<\nu<1, \nu_{3}=.755 \ldots$, and that there are exactly two. The smaller decreases to 0 , the larger increases to $\sqrt{3}$, as $\nu \rightarrow 1-$, according to his diagrammes. This has been shown by a different method from the one used here and will be published separately [4].

The results established here and in [4] are reminiscent of the corresponding phenomena for the zeros $j_{\nu k}, j_{\nu k}^{\prime}$, and $j_{\nu k}^{\prime \prime}$ of $J_{\nu}(x), J_{\nu}^{\prime}(x)$, and $J_{\nu}^{\prime \prime}(x)$, respectively.

For $\nu>0$, monotonicity of $j_{\nu k}$ was pointed out already in 1876 by L. Schläfli, for $j_{\nu k}^{\prime}$ by P. Schafheitlin in 1907, and extended, in both cases, by G. N. Watson [10, $\S 15.6$, pp. 507-510]. His method encompasses zeros of $C_{\nu}(x)=A J_{\nu}(x)+B Y_{\nu}(x), A$ and $B$ independent of $x$ and $\nu$, where $Y_{\nu}(x)$ is the Bessel function of the second kind; it covers $C_{\nu}^{\prime}(x)$ as well.

For $j_{\nu k}^{\prime \prime}, \nu>0$, monotonicity properties have been established only recently $[6,7$, $8,11]$. These state that $j_{\nu k}^{\prime \prime}$ increases with $\nu$ in $0<\nu<\infty$, for each fixed $k=1,2, \ldots$ In addition, it has been shown [5] that $j_{\nu k}^{\prime \prime}$ increases in $-1<\nu<\infty$ when $j_{\nu k}^{\prime \prime}>j_{01}=$ $2.4048 \ldots$ and, further, that when $\nu_{2}(=-0.199 \ldots)<\nu<0$, there exist precisely two zeros of $J_{\nu}^{\prime \prime}(x)$ for which $j_{\nu 1}^{\prime \prime}<j_{\nu 2}^{\prime \prime}<j_{\nu 1}$. Here $j_{\nu 1}^{\prime \prime}$ decreases to 0 while $j_{\nu 2}^{\prime \prime}$ increases to $j_{01}^{\prime \prime}=j_{11}^{\prime}=1.8411 \ldots$, as $\nu \rightarrow 0-$.

Received April 12, 1994, revised December 2, 1994.

1991 Mathematics Subject Classification: Primary 33C10; Secondary 34C10, 34L15.

Key words and phrases: Bessel functions, zeros, monotonicity, inequalities, boundary value problems, eigenvalues.

This work received partial support from the Natural Sciences and Engineering Research Council of Canada. 
These last results parallel the properties suggested by the Gray diagrammes for $j_{\nu 1}^{\prime \prime \prime}<j_{\nu 2}^{\prime \prime \prime}<\sqrt{3}, \nu_{3}<\nu<1$.

All in all, this emerging pattern would suggest, e.g., the possibility that (i) $j_{\nu k}^{(n)}$ increases in $n-1<\nu<\infty$, and (ii) for $j_{\nu k}^{(n)}>j_{\nu 1}, j_{\nu k}^{(n)}$ increases in $n-2<\nu<$ $\infty, n=1,2, \ldots$ Here $j_{\nu k}^{(n)}$ denotes the $k$-th positive zero of $J_{\nu}^{(n)}(x)$. This would exhibit yet more elegant and simply stated monotonicity properties, none trivial, in the seemingly inexhaustible number possessed by these venerable, ubiquitous, and useful functions.

A word of caution: "Monotonicity" refers to local monotonicity. This is noted already in the way Watson presents the monotonicity properties of $c_{\nu k}$ and $c_{\nu k}^{\prime}$ [10, $\S 15.6$, pp. 507-510], the zeros of $C_{\nu}(x)$ and $C_{\nu}^{\prime}(x)$, respectively. The reason is that the variation of $\nu$ brings into being new zeros, e.g., of $J_{\nu}^{\prime}(x)$ as $\nu$ increases through 0 , of $J_{\nu}^{\prime \prime}(x)$, as $\nu$ increases through $\nu_{2}=-0.199 \ldots$ and again through 1 , and of $J_{\nu}^{\prime \prime \prime}(x)$ as $\nu$ increases through $\nu_{3}=0.755 \ldots$ and again through 2 . These become the first (smallest) positive zeros, thereby causing a change in rank of all the pre-existing zeros.

Our monotonicity results will be obtained from the sign of $d j_{\nu k}^{\prime \prime \prime} / d \nu$. To develop a formula for this derivative, we shall adopt the differential equations approach successfully utilized by A. McD. Mercer [8] in his study of $d j_{\nu k}^{\prime \prime} / d \nu$, motivated by the application of that method to $d j_{\nu k}^{\prime} / d \nu$ by M. Hačik and E. Michalikova [1]. The latter refer, in turn, to a similar approach taken by M. E. Muldoon [9]. Their approach differs substantially from, and is considerably simpler than, that found in [5] and [11].

The Bessel function $J_{\nu}(x)$ is defined by the alternating series

$$
J_{\nu}(x)=\frac{1}{2^{\nu}} \sum_{k=0}^{\infty} \frac{(-1)^{k} x^{2 k+\nu}}{4^{k} k ! \Gamma(\nu+k+1)}
$$

and satisfies the differential equation

$$
x^{2} y^{\prime \prime}+x y^{\prime}+\left(x^{2}-\nu^{2}\right) y=0, \quad y=J_{\nu}(x) .
$$

Differentiating and then eliminating the $y^{\prime \prime}$ term yields an equation which will be central to the discussion below.

$$
x^{3} y^{\prime \prime \prime}+x\left(x^{2}-\nu^{2}-2\right) y^{\prime}-\left(x^{2}-3 \nu^{2}\right) y=0 .
$$

\section{Inferences from the Mittag-Leffler representation}

Various components of the proofs that $d \lambda / d \nu>0$ and of various inequalities are assisted by properties such as (2.7) of $\lambda=j^{\prime \prime \prime}$ that follow readily from the MittagLeffler type representation $[10, \S 15.41$, p. 498]

$$
\frac{J_{\nu+1}(x)}{J_{\nu}(x)}=\sum_{k=1}^{\infty} \frac{2 x}{j_{\nu k}^{2}-x^{2}}, \quad x \neq j_{\nu k} .
$$

This can be rewritten in a form appropriate to the intended applications via a standard recursion formula $[10, \S 3.2(4)$, p. 45$]$ followed by (1.3),

$$
\frac{\lambda J_{\nu+1}(\lambda)}{J_{\nu}(\lambda)}=\nu-\frac{\lambda J_{\nu}^{\prime}(\lambda)}{J_{\nu}(\lambda)}=\nu-\frac{\lambda^{2}-3 \nu^{2}}{\lambda^{2}-\nu^{2}-2}
$$

for $\lambda^{2} \neq \nu^{2}+2$. 
Thus,

$$
\sum_{k=1}^{\infty} \frac{2 \lambda}{j_{\nu k}^{2}-\lambda^{2}}=(\nu-1) \frac{\lambda^{2}-\nu^{2}+2 \nu}{\lambda^{2}-\nu^{2}-2}, \quad \lambda^{2} \neq \nu^{2}+2
$$

The requirement above that $\lambda \neq j_{\nu k}$ is a consequence of the assumption that $\lambda^{2} \neq \nu^{2}+2$ : If $\lambda$ were to be a $j_{\nu k}$, then the differential equation (1.3) would imply that

$$
\lambda\left(\lambda^{2}-\nu^{2}-2\right) y^{\prime}(\lambda)=0
$$

so that $y^{\prime}(\lambda)$ would vanish, since $\lambda^{2} \neq \nu^{2}+2$. But then $y^{\prime}(\lambda)=y(\lambda)=y\left(j_{\nu k}\right)=0$, which is impossible in view of the familiar uniqueness theorem applied to the Bessel differential equation (1.2).

Accordingly, the following inferences may be drawn from (2.3):

$$
\begin{gathered}
\text { If } \lambda^{2} \neq 3 \text { and } \nu=1, \quad \text { then } \lambda>j_{11}=3.83 \ldots, \\
\quad j_{11}^{\prime \prime \prime}=\sqrt{3}, \quad(\text { cf. also }(1.3)) . \\
\text { If } 0<\nu<1 \text {, and } \lambda<j_{\nu 1}, \quad \text { then } \lambda^{2}<\nu^{2}+2<3 . \\
\text { If } 1<\nu<2, \quad \text { then } \lambda^{2}>\nu^{2}+2>3 . \\
\text { If } \nu>2 \text { and } \lambda<j_{\nu 1}, \quad \text { then } \lambda^{2}<\nu(\nu-2) \text { or } \lambda^{2}>\nu^{2}+2 .
\end{gathered}
$$

A new (small) zero of $J_{\nu}^{\prime \prime \prime}(x)$ comes into existence as $\nu$ increases through 2 . This is analogous to the arrival of a new (small) zero of $J_{\nu}^{\prime \prime}(x)$ as $\nu$ increases through 1 [10, $\S 15.3$, p. 486]. Thus, $j_{\nu 1}^{\prime \prime \prime} \rightarrow 0$, as $\nu \rightarrow 2+$ while $j_{\nu 2}^{\prime \prime \prime 2}>\nu^{2}+2, \nu>2$.

From (2.4)-(2.8) it follows that

$$
\begin{gathered}
\lambda^{2}=\nu^{2}+2 \text { if and only if } \nu^{2}=1 \text { and } \lambda^{2}=3, \\
\text { i.e., if and only if } \lambda=j_{11}^{\prime \prime \prime} .
\end{gathered}
$$

Remarks. 1. The bound for $\lambda$ given in (2.6) can be sharpened to $\lambda^{2}<3 \nu^{2}<$ $j^{\prime 2}{ }_{\nu 1}^{2}, 0<\nu<1$, for $\lambda<j_{\nu 1}$. The bound in (2.7) can be sharpened to $\lambda^{2}>\nu^{2}+2 \nu, 1<$ $\nu<2$.

2. The inequalities (2.8) can be made more explicit. The resulting formulation, still for $\nu>2$ and $\lambda<j_{\nu 1}$, asserts that there exist two values, $\lambda_{1}, \lambda_{2}$, of $\lambda$ such that

$$
\lambda_{1}^{2}<\nu(\nu-2)<\nu^{2}+2 \nu<\lambda_{2}^{2}<j_{\nu 1}^{2}, \quad \nu>2
$$

To prove this, we note first that $j_{\nu 1}^{2}>{j^{\prime}}_{\nu 1}^{2}>\nu^{2}+2 \nu[10, \S 15.3(8)$, p. 487]. With $x^{2}=\nu^{2}+2 \nu,(1.3)$ becomes

$$
\begin{gathered}
\left(\nu^{2}+2 \nu\right)^{3 / 2} J_{\nu}^{\prime \prime \prime}\left(\sqrt{\nu^{2}+2 \nu}\right)+2\left(\nu^{2}+2 \nu\right)^{1 / 2}(\nu-1) J_{\nu}^{\prime}\left(\sqrt{\nu^{2}+2 \nu}\right) \\
+2 \nu(\nu-1) J_{\nu}\left(\sqrt{\nu^{2}+2 \nu}\right)=0 .
\end{gathered}
$$

The second and third terms are each positive for $\nu>1$ and so

$$
J_{\nu}^{\prime \prime \prime}\left(\sqrt{\nu^{2}+2 \nu}\right)<0, \quad \nu>1 .
$$


Similarly, $J_{\nu}^{\prime \prime \prime}\left(\sqrt{\nu^{2}+2}\right)<0, \nu>1$. From (1.1) it is easy to see that $J_{\nu}^{\prime \prime \prime}(0)>0, \nu>2$. Thus, there exists $\lambda_{1}$ such that $\lambda_{1}^{2}<\nu^{2}+2$. The first inequality in (2.10) now is immediate from (2.8). Further, (1.3) becomes, when $x=j_{\nu 1}$,

$$
j_{\nu 1}^{3} J_{\nu}^{\prime \prime \prime}\left(j_{\nu 1}\right)+j_{\nu 1}\left(j_{\nu 1}^{2}-\nu^{2}-2\right) J_{\nu}^{\prime}\left(j_{\nu 1}\right)=0 .
$$

The second term being negative, we have $J_{\nu}^{\prime \prime \prime}\left(j_{\nu 1}\right)>0, \nu>2$, and so the remaining inequalities in (2.10) follow.

3. It can be shown that $J_{\nu}^{\prime \prime \prime}(x)$ vanishes only twice in $0<x<j_{\nu 1}$ when $\nu>2$.

4. The upper bound in (2.6) finds a companion lower bound in (4.3).

5. Associated with (2.5) is the observation that if $\lambda \neq \sqrt{3}$, then $\lambda^{2} \neq \nu^{2}+2$.

6. Inequalities (2.6)-(2.8) are used below. Stronger inequalities, not needed here, are established in $[4, \S 5]$. Alternatively, one can strengthen them also by using the inequalities for $J_{\nu+1}(\lambda) / J_{\nu}(\lambda), 0<\lambda<j_{\nu 1}$, found in [2].

\section{The derivative of $j_{\nu k}^{\prime \prime \prime}$ with respect to the order $\nu, k$ fixed}

Here we obtain representations (3.4), (3.5), and (3.7) for $d j_{\nu k}^{\prime \prime \prime} / d \nu, k=1,2, \ldots$, from which monotonicity properties of $j_{\nu k}^{\prime \prime \prime}$ will be inferred in subsequent sections. For typographical convenience, we write $\lambda=\lambda_{\nu}=j_{\nu k}^{\prime \prime \prime}$, with the understanding that this is generic, that $\lambda$ is a function of $\nu$ and that $k$ is fixed, $k=1,2, \ldots$ As usual, $j_{\nu k}^{\prime \prime \prime}$ is the $k$-th positive zero of $J_{\nu}^{\prime \prime \prime}(x)$.

The boundary-value problem which arises in this connection is, for $y(x)=J_{\nu}(\lambda x)$,

$$
\left(x y^{\prime}\right)^{\prime}=\frac{\nu^{2}}{x} y-\lambda^{2} x y
$$

with $y(0)=0, y^{\prime \prime \prime}(1)=0$.

The stated boundary conditions can be written equivalently as

$$
y(0)=0, \quad y^{\prime}(1)=\frac{3 \nu^{2}-\lambda^{2}}{\nu^{2}+2-\lambda^{2}} y(1), \quad \lambda^{2} \neq \nu^{2}+2 .
$$

This is a consequence of (1.3).

With $\lambda^{2} \neq \nu^{2}+2$, i.e., $\lambda \neq j_{11}^{\prime \prime \prime}=\sqrt{3}$,

$$
y^{\prime}(1)=\lambda J_{\nu}^{\prime}(\lambda)=\frac{\lambda^{2}-3 \nu^{2}}{\lambda^{2}-\nu^{2}-2} J_{\nu}(\lambda)=\frac{\lambda^{2}-3 \nu^{2}}{\lambda^{2}-\nu^{2}-2} y(1) .
$$

The device employed in [1] and [8] is useful here as well. That is, from (3.1) we obtain the two equations

$$
\left(x y^{\prime}\right)^{\prime}=\frac{\nu^{2}}{x} y-\Lambda x y
$$

and

$$
\left(x v^{\prime}\right)^{\prime}=\frac{(\nu+\varepsilon)^{2}}{x} y-\Gamma x v,
$$

where $\Lambda=\lambda^{2}$ and $\Gamma$ is the corresponding parameter associated with the order $\nu+\varepsilon$ and $v$ is the new solution. 
Continuing, multiplying the first equation by $v$, the second by $y$, subtracting and integrating over $(0,1)$, we have

$$
y^{\prime}(1) v(1)-v^{\prime}(1) y(1)=\left[\nu^{2}-(\nu+\varepsilon)^{2}\right] \int_{0}^{1} \frac{y v}{x} d x-(\Lambda-\Gamma) \int_{0}^{1} x y v d x .
$$

This becomes, in view of (3.2) and division by $\varepsilon$,

$$
\begin{aligned}
& y(1) v(1) \varepsilon^{-1}\left[\frac{3 \nu^{2}-\Lambda}{\nu^{2}+2-\Lambda}-\frac{3(\nu+\varepsilon)^{2}-\Gamma}{(\nu+\varepsilon)^{2}+2-\Gamma}\right] \\
& =\frac{\left[\nu^{2}-(\nu+\varepsilon)^{2}\right]}{\varepsilon} \int_{0}^{1} \frac{y v}{x} d x-\frac{\Lambda-\Gamma}{\varepsilon} \int_{0}^{1} x y v d x .
\end{aligned}
$$

Letting $\varepsilon \rightarrow 0$ and multiplying by -1 , we obtain

$$
y^{2}(1) \frac{d}{d \nu}\left\{\frac{3 \nu^{2}-\Lambda}{\nu^{2}+2-\Lambda}\right\}=2 \nu \int_{0}^{1} \frac{y^{2}(x)}{x} d x-\frac{d \Lambda}{d \nu} \int_{0}^{1} x y^{2}(x) d x .
$$

This can be written, provided $\lambda^{2} \neq \nu^{2}+2$, i.e., for $\lambda \neq j_{11}^{\prime \prime \prime}$, as

$$
\begin{gathered}
\left\{\frac{1}{\lambda^{2}} \int_{0}^{\lambda} t J_{\nu}^{2}(t) d t+\frac{2\left(\nu^{2}-1\right)}{\left(\nu^{2}+2-\lambda^{2}\right)^{2}} J_{\nu}^{2}(\lambda)\right\} 2 \lambda \frac{d \lambda}{d \nu} \\
=2 \nu \int_{0}^{\lambda} \frac{J_{\nu}^{2}(t)}{t} d t-\frac{4 \nu\left(3-\lambda^{2}\right)}{\left(\nu^{2}+2-\lambda^{2}\right)^{2}} J_{\nu}^{2}(\lambda) .
\end{gathered}
$$

The transition to (3.4) follows on carrying out the indicated differentiation (bearing in mind that $\Lambda$ is a function of $\nu$ ), collecting the terms involving $d \Lambda / d \nu$, and finally replacing $\Lambda$ by $\lambda^{2}$.

This is the formula which we shall use to determine the sign of $d \lambda / d \nu$, and hence the monotonicity of $\lambda$, as a function of $\nu$, for appropriate $\nu$ and $\lambda$. It can be written in alternative forms. For example, the coefficient of $d \lambda / d \nu$ can be transformed by using $[10, \S 5.11(11)$, p. 135$]$ in the form

$$
\frac{1}{\lambda^{2}} \int_{0}^{\lambda} t J_{\nu}^{2}(t) d t=\frac{1}{2}\left\{\left(1-\frac{\nu^{2}}{\lambda^{2}}\right) J_{\nu}^{2}(\lambda)+\left[J_{\nu}^{\prime}(\lambda)\right]^{2}\right\}
$$

and then replacing $J_{\nu}^{\prime}(\lambda)$ by means of (3.3). This done, (3.4) becomes, for $\lambda^{2} \neq \nu^{2}+2$, i.e., for $\lambda \neq j_{11}^{\prime \prime \prime}=\sqrt{3}$,

$$
\frac{J_{\nu}^{2}(\lambda)}{\lambda^{2}\left(\nu^{2}+2-\lambda^{2}\right)^{2}} Q_{\nu}(\lambda) \frac{d \lambda}{d \nu}=2 \nu \int_{0}^{\lambda} \frac{J_{\nu}^{2}(t)}{t} d t-\frac{4 \nu\left(3-\lambda^{2}\right)}{\left(\nu^{2}+2-\lambda^{2}\right)^{2}} J_{\nu}^{2}(\lambda),
$$

where the polynomial $Q_{\nu}(\lambda)$ is defined by

$$
Q_{\nu}(\lambda)=\lambda^{6}-\left(3 \nu^{2}+1\right) \lambda^{4}+3 \nu^{2}\left(\nu^{2}+2\right) \lambda^{2}-\nu^{2}\left(\nu^{2}-1\right)\left(\nu^{2}-4\right),
$$

which, reverting to (3.4), is positive for all $\nu \geq 1$.

The right-hand side of (3.5) can also be written otherwise, since [10, $§ 5.5$ (5), p. 152]

$$
2 \nu \int_{0}^{x} \frac{J_{\nu}^{2}(t)}{t} d t=J_{\nu}^{2}(x)+2 \sum_{k=1}^{\infty} J_{\nu+k}^{2}(x)
$$


which, applied to (3.5), gives, again for $\lambda^{2} \neq \nu^{2}+2$,

$$
\begin{aligned}
& \frac{J_{\nu}^{2}(\lambda)}{\lambda\left(\nu^{2}+2-\lambda^{2}\right)^{2}} Q_{\nu}(\lambda) \frac{d \lambda}{d \nu} \\
& \quad=\left[1-\frac{4 \nu\left(3-\lambda^{2}\right)}{\left(\nu^{2}+2-\lambda^{2}\right)^{2}}\right] J_{\nu}^{2}(\lambda)+2 \sum_{k=1}^{\infty} J_{\nu+k}^{2}(\lambda) .
\end{aligned}
$$

This can be transformed still further, say by first using recursion formulae to write $J_{\nu+1}(\lambda)$ and $J_{\nu+2}(\lambda)$ in terms of $J_{\nu}(\lambda)$ and $J_{\nu}^{\prime}(\lambda)$ and then by expressing $J_{\nu}^{\prime}(\lambda)$ in terms of $J_{\nu}(\lambda)$ via (3.3).

\section{Positivity of the coefficient of $d \lambda / d \nu$}

We prove here that the coefficient of $d \lambda / d \nu$ in (3.4), and hence in (3.5) and (3.7), is positive, for all $\nu>0$, with $\lambda=j_{\nu k}^{\prime \prime \prime}$.

For $\nu \geq 1$, this is obvious in (3.4) and hence must be true as well for (3.5) and (3.7), as rewritings of (3.4).

For $0<\nu<1$, our assertion is equivalent to

$$
Q_{\nu}(\lambda)>0
$$

an inequality which is valid also for $\nu \geq 1$, where $Q_{\nu}(\lambda)$ is defined by (3.6).

This we consider in two steps: (a) $\lambda^{2} \geq 3$ and (b) $\lambda^{2}<3$. Common to both is the need to establish that $Q_{\nu}(x)$ is an increasing function of $x$ for fixed $\nu>0$, in applicable intervals.

We note that $Q_{\nu}(x)$ increases for all $x>0$ when $\frac{1}{12} \leq \nu^{2}<1$, and that when $\nu^{2} \leq \frac{1}{12}$ it increases for

$$
x^{2}>\frac{1}{3}\left[3 \nu^{2}+1+\sqrt{1-12 \nu^{2}}\right]:=\alpha_{\nu}
$$

as is clear from its derivative $Q_{\nu}^{\prime}(x)$.

But $\alpha_{\nu} \leq \frac{2}{3}$, so that in case (a),

$$
Q_{\nu}(\lambda) \geq Q_{\nu}(\sqrt{3})=-\nu^{6}+14 \nu^{4}-13 \nu^{2}+18>14.87>0, \quad 0<\nu<1,
$$

since here $\lambda \geq \sqrt{3}$.

For case (b), we can use again the property just noted that $Q_{\nu}(x)$ increases for all $x>0$ when $\frac{1}{12} \leq \nu^{2}<1$. This is because

$$
\lambda>j_{\nu 1}>j_{01}=2.4048 \ldots>\sqrt{3}, \quad 0<\nu \leq \nu_{0}=0.7552027 \ldots,
$$

as we shall establish a bit later.

We need a lower bound for $\lambda$ in order to make use of the monotonicity of $Q_{\nu}(x)$. It is enough to know that

$$
\lambda^{2}>1-\nu, \quad \nu>0
$$

also verified a bit later.

Thus, from (4.3) and (3.6),

$$
Q_{\nu}(\lambda)>Q_{\nu}(\sqrt{1-\nu})=\nu(1-\nu)\left(\nu^{4}+\nu^{3}-\nu^{2}+3 \nu-1\right)
$$

which is positive for $\frac{1}{2}<\nu<1$ and hence all the more so for the interval $\nu_{0}<\nu<1$, relevant here. 
Now we prove (4.2) and (4.3). For (4.2) we use the third derived series for $J_{\nu}(x)$, i.e.,

$$
f_{\nu}(x):=2^{\nu} \Gamma(\nu+1) x^{3-\nu} J_{\nu}^{\prime \prime \prime}(x)=\sum_{k=0}^{\infty} \frac{(-1)^{k}(\nu+2 k)(\nu+2 k-1)(\nu+2 k-2) x^{2 k}}{4^{k} k !(\nu+k) \cdots(\nu+1)},
$$

where the constant term is understood to be $\nu(\nu-1)(\nu-2)$.

From this alternating series it is easy to see that, for $x^{2} \leq \nu^{2}+2$,

$$
\begin{aligned}
384 f_{\nu}(x)> & 384 \nu(\nu-1)(\nu-2)-96 \nu(\nu+2) x^{2} \\
+ & \frac{12(\nu+3)(\nu+4) x^{4}}{\nu+1}-\frac{(\nu+4)(\nu+5)(\nu+6) x^{6}}{(\nu+1)(\nu+2)(\nu+3)}:=p_{\nu}(x) .
\end{aligned}
$$

The polynomial $p_{\nu}(x)$, a cubic in $x^{2}$, reaches its minimum (in $x^{2} \leq \nu^{2}+2$ ) when

$$
x_{0}^{2}=\frac{4(\nu+2)\left[(\nu+3)^{2}(\nu+4)-A_{\nu} \sqrt{(\nu+3)(\nu+4)}\right]}{(\nu+4)(\nu+5)(\nu+6)},
$$

where

$$
A_{\nu}=\sqrt{-\nu^{4}-11 \nu^{3}-19 \nu^{2}+75 \nu+108}
$$

The sign of $p_{\nu}(x)$ is preserved on multiplication by

$$
(\nu+1)(\nu+2)(\nu+3)(\nu+4)^{2}(\nu+5)^{2}(\nu+6)^{2} .
$$

This done, we evaluate the resulting polynomial at its minimum $x=x_{0}$. Then

$$
\begin{aligned}
\frac{(\nu+1)(\nu+2)(\nu+3)(\nu+4)^{2}(\nu+5)^{2}(\nu+6)^{2}}{384(\nu+2)(\nu+3)(\nu+4)} p_{\nu}\left(x_{0}\right) \\
=\frac{(\nu+1)(\nu+4)(\nu+5)^{2}(\nu+6)^{2}}{384} p_{\nu}\left(x_{0}\right) \\
=(\nu+4)\left(\nu^{8}+17 \nu^{7}+44 \nu^{6}-692 \nu^{5}-4785 \nu^{4}-7977 \nu^{3}+5796 \nu^{2}\right. \\
\quad+13500 \nu+3888)-(\nu+2)^{2}(\nu+3)^{1 / 2}(\nu+4)^{1 / 2} A_{\nu}^{3} \\
:=p_{\nu 1}\left(x_{0}\right)-p_{\nu 2}\left(x_{0}\right) .
\end{aligned}
$$

In $0<\nu<1$, each term is positive, so that their difference is positive when and only when the difference of their squares is positive. But

$$
p_{\nu 1}^{2}\left(x_{0}\right)-p_{\nu 2}^{2}\left(x_{0}\right)=2 \nu(\nu+1)(\nu+5)^{2}(\nu+6)^{2} F(\nu),
$$

where

$$
\begin{aligned}
F(\nu)=\nu^{11} & +18 \nu^{10}+55 \nu^{9}-616 \nu^{8}-2867 \nu^{7} \\
& +13400 \nu^{6}+90471 \nu^{5}+68054 \nu^{4}-271692 \nu^{3} \\
& -218232 \nu^{2}+134784 \nu+93312 .
\end{aligned}
$$

The polynomial $F(\nu)$ is positive for $0 \leq \nu \leq \nu_{0}$, where $\nu_{0}=.7552027 \ldots$.

Thus, $J_{\nu}^{\prime \prime \prime}(x)>0, x^{2} \leq \nu^{2}+2$, when $0 \leq \nu \leq \nu_{0}$. In view of (2.6), this concludes the proof of (4.2). 
The proof of (4.3) is simpler. With $x^{2} \leq 1-\nu$, we note that

$$
\begin{aligned}
f_{\nu}(x) & >\nu(\nu-1)(\nu-2)-\frac{\nu(\nu+2) x^{2}}{4} \\
& \geq \nu(\nu-1)(\nu-2)-\frac{1}{4} \nu(\nu+2)(1-\nu) \\
& =\frac{1}{4} \nu(1-\nu)(6-5 \nu)>0,0<\nu<1,
\end{aligned}
$$

so that $J_{\nu}^{\prime \prime \prime}(x)>0, x^{2} \leq 1-\nu$, verifying (4.3).

Remark. We thank Donald H. Pelletier for his help with the calculations included in (4.5) and (4.6).

\section{Local monotonicity of $j_{\nu k}^{\prime \prime \prime}$}

The main local monotonicity properties are obvious from (3.5) and (4.1).

Theorem 5.1. If $\lambda^{2} \geq 3$ and $\nu>0$, then $d \lambda / d \nu>0, \lambda \neq j_{11}^{\prime \prime \prime}$.

The hypothesis that $\lambda^{2} \geq 3$ can be dropped from Theorem 5.1, provided we restrict $\nu$ to be at least 1 .

Theorem 5.2. If $\nu \geq 1$, then $d \lambda / d \nu>0$.

Proof. Here we consider first $1<\nu \leq 2$. For these, (2.7) shows that $\lambda^{2}>\nu^{2}+2>3$. Thus, such $\nu$ are already covered by Theorem 5.1. It remains to consider $\nu>2$ (and these $\nu$ only for $\lambda^{2}<3$, again in view of Theorem 5.1).

The coefficient of $d \lambda / d \nu$ in (3.4), and hence also in (3.7), is positive ( $\S 4)$. Proof will be complete on showing that the right-hand side of (3.7) is positive when $\nu>2$ and $\lambda^{2}<3$, i.e., that

$$
\frac{4 \nu\left(3-\lambda^{2}\right)}{\left(\nu^{2}+2-\lambda^{2}\right)^{2}} \leq 1, \quad \nu>2 .
$$

In fact,

$$
\frac{4 \nu\left(3-\lambda^{2}\right)}{\left(\nu^{2}+2-\lambda^{2}\right)^{2}}<\frac{2}{3}, \quad \nu>2 .
$$

This holds if

$$
\lambda^{4}-2\left(\nu^{2}-3 \nu+2\right) \lambda^{2}+\nu^{4}+4 \nu^{2}-18 \nu+4>0, \quad \nu>2 .
$$

The discriminant of this quadratic in $\lambda^{2}$ is

$$
-12 \nu(2 \nu+1)(\nu-2)<0, \quad \nu>2,
$$

so that the quadratic has no real roots for $\nu>2$. Being eventually positive, then, the quadratic in $\lambda^{2}$ is always positive and the requisite inequality is verified, completing the proof of Theorem 5.2. 
Remark. Theorem 5.2 holds also for $0<\nu<\nu_{0}$. This is clear from (4.2) and Theorem 5.1. However, the restriction $\nu \geq 1$ in Theorem 5.2 cannot be dropped entirely. As Alfred Gray's diagrammes exhibit and [4] establishes, $j_{\nu 1}^{\prime \prime \prime}$ decreases although $j_{\nu 2}^{\prime \prime \prime}$ increases in $\nu_{3}<\nu<1$.

Calculations of these quantities, motivated by the Gray diagrammes, were made before the work for [4] was done. Some were done as demonstrations at the August 1993 mathematics meeting in Vancouver, on "Derive", by David R. Stoutemyer (Soft Warehouse, Honolulu), and by S. Adams and Keith O. Geddes using "Maple V, Release 2" (Waterloo, Ontario, Canada), later redone by Martin Muldoon using "Maple V, Release 1".

These calculations give, as would be expected, a larger value than $\nu_{0}$, denoted by $\nu_{3}$ above. It is perhaps surprising that the values are so close. We have $\nu_{0}=$ $0.7552027 \ldots$; the Maple programme extends the validity of $(4.2)$ to $\nu=0.75558 \ldots$. For $\nu=0.75558 \ldots, J_{\nu}^{\prime \prime \prime}(x)$ has a double zero which, pursuant to [3, (2.5)], occurs at $x=0.95939 \ldots$.

\section{References}

1. M. Hačik and E. Michalikova, A note on monotonicity of zeros of Bessel functions as functions of order, Práce Studia Vysokej Skoly Doprovy a Spojov Žiline, Ser. Math.-Fyzik 1989, 7-13.

2. E. K. Ifantis and P. D. Siafarikas, Inequalities involving Bessel and modified Bessel functions, J. Math. Anal. Appl. 147 (1990), 214-227.

3. M. K. Kerimov and S. L. Skorokhodov, On nultiple zeros of derivatives of Bessel's cylindrical functions, Soviet Math. Dokl. 33 (1986), 65 -653.

4. L. Lorch, The zeros of the third derivative of Bessel functions of order less than one, Math. Anal. Appl. (to appear).

5. L. Lorch, M. E. Muldoon, and P. Szego, Inj ection points of Bessel functions of negative order, Canadian J. Math. 43 (1991), 1309-1322.

6. L. Lorch and P. Szego, On the points of inflection of Bessel functions of positive order I, Canadian J. Math. 42 (1990), 933-948; ibid., 1132.

7. __ Further on the points of inflection of Bessel functions, Canadian Math. Bull. (to appear).

8. A. McD. Mercer, The zeros of $a z^{2} J_{\nu}^{\prime \prime}(z)+b z J_{\nu}^{\prime}(z)+c J_{\nu}(z)$ as functions of order, Intl. J. Math. and Math. Sci. 15 (1992), 319-322.

9. M. E. Muldoon, On the zeros of a cross-product of Bessel functions of different orders, Z. Angew. Math. Mech. 59 (1979), 272-273.

10. G. N. Watson, A Treatise on the Theory of Bessel Functions, 2nd ed., The University Press Cambridge, 1944.

11. R. Wong and T. Lang, On the points of inflection of Bessel functions of positive order II. Canadian J. Math. 43 (1991), 628-651.

Department of Mathematics, York University, 4700 Keele Street, North York, Ontario, CANADA M3J 1P3

E-mail: lorch@mathstat.yorku.ca

75 Glen Eyrie Avenue, Apt. 19, San Jose, California 95125, U.S.A. 